\title{
Polyaniline integrated carbon nanohorn: A superior electrode materials for advanced energy storage
}

\author{
S. Maiti, B. B. Khatua* \\ Materials Science Centre, Indian Institute of Technology, 721302 Kharagpur, India
}

Received 18 April 2014; accepted in revised form 29 June 2014

\begin{abstract}
Fiber-like polyaniline (PANI)/carbon nanohorn (CNH) composites (PACN composites) were prepared as electrode materials for supercapacitor by simple method that involves in-situ polymerization of aniline in the presence of $\mathrm{CNH}$ in acidic $(\mathrm{HCl})$ medium with noteworthy electrochemical performances. Thus, the prepared PACN composites show high specific capacitance value of $\approx 834 \mathrm{~F} / \mathrm{g}$ at $5 \mathrm{mV} / \mathrm{s}$ scan rate compared to $\approx 231 \mathrm{~F} / \mathrm{g}$ for pure PANI and CNH $(\approx 145 \mathrm{~F} / \mathrm{g})$ at same scan rate of $5 \mathrm{mV} / \mathrm{s}$. CNHs are homogeneously dispersed throughout the matrix and coated successfully. Thus, it provides more active sites for nucleation and electron transfer path. In addition, the composites show high electrical conductivity in the order of $\approx 6.7 \cdot 10^{-2} \mathrm{~S} \cdot \mathrm{cm}^{-1}$ which indicates the formation of continuous interconnected conducting network path in the PACN composites. Morphological study of the PACN composites was carried out by high resolution transmission electron microscopy (HRTEM) and field emission scanning electron microscopy (FESEM).
\end{abstract}

Keywords: nanocomposites, supercapacitor, polyaniline, carbon nanohorn, electrical conductivity

\section{Introduction}

Presently, various motor vehicles and portable electronic devices have increased the requirement of energy storage devices. Thus, high performance supercapacitors and different batteries (Li-ion, $\mathrm{Pb}$ acetate etc) have been attracted in the various fields of applications. Recently, supercapacitors have been a major source for huge amount of energy in a very small period of time and make them essential for surge power delivery. Supercapacitors have been considered most promising materials for alternative energy sources in the different device and electronics applications due to their rapid charging-discharging rates, high power density and long cycle life (>100 000 cycles) [1-3]. Thus, they have been taken as more important components for all electric cars and cars based on fuel cells like hydrogen fuel cell and direct methanol fuel cell (DMFC) in the modern generation $[4,5]$. In general, supercapacitors have been greatly used as complementary devices to different fuel cells and batteries due to their high energy densities but unable to supply a high power during short time period. The electrochemical capacitors have high-power sources and have been used also for digital communication devices [6-9]. Depending on the charge-storage mechanism, supercapacitors have been categorized into two different types [ 10 , 11]: (a) electrical double-layer capacitors (EDLCs) for carbon-based active materials with high surface area, the capacitance value is obtained due to the charge separation at the electrode-electrolyte interface. The charge storage mechanism for EDLCs is of non-Faradic type; electrical charge builds up at the electrode-electrolyte interface. Generally, EDLCs have high power density but major disadvantage is low capacitance and low rate capability. In the case of EDLCs, the charge can be stored electrostatically or non-Faradaically (in case of carbon nanomateri-

\footnotetext{
${ }^{*}$ Corresponding author, e-mail: khatuabb@matsc.iitkgp.ernet.in (C) BME-PT
} 
als) like conventional capacitors and charge transfer does not occur between the electrode and the electrolyte, and (b) others are pseudocapacitors which develop fast and reversible surface or near surface reactions for charge storage. For pseudocapacitors, electrode materials may be metal oxides $(\mathrm{NiO}$, $\mathrm{MnO}_{2}$ etc) or conducting polymers and Faradic reactions occur at the electrode-electrolyte interface. The charge is stored Faradaically through charge transfer between electrolyte and electrode. This process is completed through electro-sorption, reduction-oxidation reactions, and intercalation processes. Among them, pseudo-capacitors could be the source of higher specific capacitance. However, major drawback of pseudo-capacitors is that they are very costly and show low conductivity. Hence, the energy storage performance of the capacitors needs to be improved for their broad potential applications under the current requirements. The high electrical conductivity, high surface area and a fast cation intercalation/de-intercalation process play a crucial role to obtain high energy and power densities for capacitors. In the case of carbon-based EDLCs, the charge storage mechanism occurs via electrostatic forces and fast faradaic redox reactions are the reason behind the storage mechanism for pseudo-capacitors.

Transition metal oxides play a key role as potential electrode materials for the preparation of supercapacitors due to their charge storage mechanisms $[12,13]$. Among the different metal oxides, $\mathrm{RuO}_{2}$ shows high capacitance value for its redox transitions. However, the major problem of Ru metal is its high cost which reduces its commercial value as a potential electrode material. Currently, the preparation of cost-effective electrode materials has become great challenge for supercapacitor applications. Cheap metal oxides such as oxides of $\mathrm{Co}, \mathrm{Ni}, \mathrm{Mn}, \mathrm{In}$, $\mathrm{Fe}, \mathrm{Sn}, \mathrm{Pb}[14,15]$ and so forth are being investigated to improve the capacitance value of the capacitors. The conducting polymers are also used as electrode materials for supercapacitors for its excellent electrochemical properties and low cost. Among the several conducting polymers such as polyaniline (PANI), polythiophene (PTH), polypyrrole (PPy) and their derivatives, PANI has been considered more useful material for supercapacitors due to its redox reversibility, high electrical conductivity, easy synthesis procedure, good environmental stability and of course its low cost [16]. However, chemically synthesized electrically conducting polymers are generally powdery and behave like an insulating material in their undoped states. Thus, carbon-based nanomaterials have been considered most promising materials for supercapacitors due to their high surface area, high aspect ratio, nano-size and very high electrical conductivity [17]. In this regard, different carbon based nanomaterials such as carbon nanotube (CNT), graphene and carbon nanohorn $(\mathrm{CNH})$ have been chosen as substrate for metal oxide nanoparticles or conducting polymers for supercapacitor applications. This carbon based conducting nanomaterials help to enhance the electron transfer rate during Faradaic charge transfer reactions in the composites and increase the value of specific capacitance [18]. Single-walled carbon nanohorns (SWCNHs) are dahlia flower like spherical superstructure of aggregated nano-sized graphitic tubes [19-21]. Due to the high surface area, considerable internal nanospace, excellent porosity and high electrical conductivity, they have been broadly used in the various filed of applications such as supercapacitors, hydrogen storage, fuel cells, drug delivery and biosensors, etc. Moreover, CNHs possess high purity and also high absorbability compared with others carbon based nanomaterials due to their large surface area, high aspect ratio and inherent micropores at interstitial sites.

Several research groups have been developed PANI composites based on metal oxides, graphene or $\mathrm{CNH}$ for supercapacitors. For instance, Wu et al. [22] showed the large electrochemical capacitance of $\approx 210 \mathrm{~F} / \mathrm{g}$ at a discharge rate of $0.3 \mathrm{~A} / \mathrm{g}$ in composite films of chemically converted graphene (CCG) and polyaniline nanofibers (PANI-NFs) prepared by vacuum filtration of the mixed dispersions of both the components. Deshmukh and Shelke [23] synthesized $\mathrm{Fe}_{3} \mathrm{O}_{4}$-SWCNH nanocomposites and reported specific capacitance value of the nanocomposites was $\approx 377 \mathrm{~F} / \mathrm{g}$ at current density of $1 \mathrm{~A} / \mathrm{g}$. Wang et al. [24] prepared one-dimensional and layered parallel folding of cobalt oxalate nanostructures and reported the maximum specific capacitance of $\approx 202.5 \mathrm{~F} / \mathrm{g}$ at a current density of $1 \mathrm{~A} / \mathrm{g}$ with a voltage window from 0 to $0.40 \mathrm{~V}$. Kumar et al. [25] have prepared conducting polyaniline-grafted reduced graphene oxide (PANi-g-rGO) composite via acyl chemistry and reported the capacitance of $\approx 250 \mathrm{~F} / \mathrm{g}$ with electrical conductivity as high as $\approx 8.66 \mathrm{~S} / \mathrm{cm}$.

In this study, we have prepared PACN composites through cost effective method which involves in- 
situ polymerization of aniline in the presence of $\mathrm{CNH}$. Thus, prepared PACN composites show very high specific capacitance value $(\approx 834 \mathrm{~F} / \mathrm{g})$ at scan rate of $5 \mathrm{mV} / \mathrm{s}$ which is higher than the specific capacitance $(\approx 231 \mathrm{~F} / \mathrm{g}$ at $5 \mathrm{mV} / \mathrm{s})$ of pure PANI and $\mathrm{CNH}(\approx 145 \mathrm{~F} / \mathrm{g})$ at same scan rate of $5 \mathrm{mV} / \mathrm{s}$. So, specific capacitance value of the pure PANI is greatly affected in the presence of $\mathrm{CNH}$ in the PACN composites. Due to homogeneous coating of conducting $\mathrm{CNH}$ in the PACN composites, it forms a conducting electron transfer path and provides more active sites for nucleation [26] and increases the capacitance value of the composites. Additionally, the synthesized PACN composites showed electrically conducting behavior and obtained electrical conductivity of the composites is $\approx 6.7 \cdot 10^{-2} \mathrm{~S} / \mathrm{cm}$ which indicates the possible formation of conducting network structure in the PACN composites.

\section{Experimental}

\subsection{Materials details}

Aniline, hydrochloric acid ( $\mathrm{HCl})$ and potassium chloride were purchased from Merck, Germany. Single-layer carbon nanohorn $(\mathrm{CNH}$, carbon purity: $>99 \%$, horn diameter: $3 \sim 5 \mathrm{~nm}$, horn length: 30 50 nm, cluster diameter: $60 \sim 120 \mathrm{~nm}$, density: $1.1 \mathrm{~g} / \mathrm{cm}^{3}$, surface area: $250 \sim 300 \mathrm{~m}^{2} / \mathrm{g}$ ) was procured from J. K. Impex, Mumbai, India. Cetyl trimethylammonium bromide (CTAB) and ammonium persulfate (APS) $\left[\left(\mathrm{NH}_{4}\right)_{2} \mathrm{~S}_{2} \mathrm{O}_{8}\right]$ were purchased from Loba Chemie Pvt. Ltd. India.

\subsection{Preparation of the PACN composites}

$100 \mathrm{~mL}$ of $1.5(\mathrm{M}) \mathrm{HCl}$ was taken in a $500 \mathrm{~mL}$ beaker and $30 \mathrm{mg}$ of $\mathrm{CNH}$ and $\mathrm{CTAB}$ were added to this acid solution. Then, the mixture was ultra-sonicated for $1 \mathrm{~h}$ for homogeneous dispersion of $\mathrm{CNH}$ in the acid media, followed by vigorous stirring for $30 \mathrm{~min}$ with magnetic stirrer at room temperature. After that, $1 \mathrm{~mL}$ aniline was added to this acidic solution of $\mathrm{CNH}$ and continued the stirring for another $10 \mathrm{~min}$. Furthermore, ammonium persulfate (APS) solution (which was prepared through dissolving $2 \mathrm{mg}$ APS in $100 \mathrm{~mL} 1.5(\mathrm{M}) \mathrm{HCl}$ ) was added into the reaction mixture as an oxidizing agent for polymerization of aniline and the stirring was continued. After $4 \mathrm{~h}$ of stirring, the solution became green and precipitation was observed. Then, the green solution was filtered and washed with de-ionized (DI) water for several times to make neutral solution. Thus, the obtained residue was air dried for $24 \mathrm{~h}$ for further characterizations. Polyaniline (PANI) was also prepared by the same procedure in the presence of CTAB.

\section{Characterizations}

\subsection{Field emission scanning electron microscope (FESEM)}

The morphology of the PACN composites was studied using PACN pellets (prepared by pressing at room temperature) through FESEM (FE-SEM, Carl ZeissSUPRA $^{\text {TM }} 40$ ), with an accelerating voltage of $5 \mathrm{kV}$. The samples were coated with a thin of layer gold (approx $\sim 5 \mathrm{~nm}$ ) to avoid the electrical charging during scanning. This gold coated composites sample was scanned in the vacuum order of $10^{-4}$ to $10^{-6} \mathrm{~mm} \mathrm{Hg}$.

\subsection{High resolution transmission electron microscope (HRTEM)}

The TEM analysis of the PACN composites was carried out by HRTEM (HRTEM, JEM-2100, JEOL, JAPAN), operated at an accelerating voltage of $200 \mathrm{kV}$. A small amount of the PACN composites was dispersed in acetone through sonication. Then, the dispersed suspension of PACN composites was dropped on the copper $(\mathrm{Cu})$ grid for HRTEM analysis.

\subsection{Electrochemical characterization}

Electrochemical analysis of as-synthesized PACN composites, pure PANI and $\mathrm{CNH}$ was performed by cyclic voltammetry $(\mathrm{CV})$ with a $\mathrm{CH}$ instrument electrochemical analyzer. A three-electrode system was employed for all measurements where PACN composites coated glassy carbon electrode, Pt wire and $\mathrm{Ag} / \mathrm{AgCl}$ served as working, counter and reference electrodes, respectively. Cyclic voltammogram was recorded at different scan rates from $5 \mathrm{mV} / \mathrm{s}$ to $100 \mathrm{mV} / \mathrm{s}$ within potential windows from -0.4 to $+0.8 \mathrm{~V}$ in $1(\mathrm{M}) \mathrm{KCl}$.

The electrochemical impedance spectroscopic (EIS) measurement was carried out in the frequency range of $100 \mathrm{mHz}-10 \mathrm{MHz}$. The specific capacitances $\left(C_{\mathrm{sp}}\right)$ of PACN composites at different scan rates were calculated by using Equation (1) [27]:

$C_{\text {sp }}=\frac{\int I \mathrm{~d} V}{v m V}$

where $I$ is the response current density, $V$ is the potential $[\mathrm{V}], v$ is the potential scan rate $[\mathrm{mV} / \mathrm{s}]$ and 
$m$ is the mass of the PACN composite in the electrodes.

\subsection{Electrical conductivity}

The DC electrical conductivity of the PACN composites was measured by four probe method. The electrical conductivity of the samples was calculated with the help of Equations (2) and (3) [28]:

Resistivity $(\rho)[\Omega \cdot \mathrm{cm}]=\frac{\pi t}{\operatorname{In} \cdot 2 \frac{V}{I}}$

Conductivity $(\sigma)\left[\mathrm{S} \cdot \mathrm{cm}^{-1}\right]=\frac{1}{\rho}$

where $V$ is the measured voltage, $I$ is the applied current. The thickness of the samples is represented by $t$. Minimum of five tests were performed for each specimen and the data was averaged.

The frequency dependent AC conductivity of the PACN composites (disc type sample with thickness $0.3 \mathrm{~cm}$ and area $1.88 \cdot 10^{-1} \mathrm{~cm}^{2}$ ) were obtained using a computer controlled precision impedance analyzer (Agilent 4294A) by applying an alternating electric field (amplitude 1000 volt) across the sample cell in the frequency region of $50 \mathrm{~Hz}$ to $1 \mathrm{MHz}$. A parallel plate configuration was used for all the electrical measurements. A sample holder using Pt probe was used for all the electrical measurements.

The parameters like dielectric permittivity $\left(\varepsilon^{\prime}\right)$ and dielectric loss tangent $(\tan \delta)$ were obtained as a function of frequency. The AC conductivity $\left(\sigma_{\mathrm{ac}}\right)$ was calculated from the dielectric data using the Equation (4):

$\sigma_{\mathrm{ac}}=\omega \varepsilon_{0} \varepsilon^{\prime} \tan \delta$

where $\omega$ is equal to $2 \pi f$ ( $f$ is the frequency), and $\varepsilon_{0}$ is vacuum permittivity. The dielectric permittivity $\left(\varepsilon^{\prime}\right)$ was determined with Equation (5):

$\varepsilon^{\prime}=\frac{C_{\mathrm{p}}}{C_{0}}$

where $C_{\mathrm{p}}$ is the observed capacitance of the sample (in parallel mode), and $C_{0}$ is the capacitance of the cell. The value of $C_{0}$ was calculated using the area $(A)$ and thickness $(d)$ of the sample, following the Equation (6):

$C_{0} \approx \frac{\varepsilon_{0} \cdot A}{d}$

\section{Results and discussion}

\subsection{Electrochemical analysis}

\subsubsection{Cyclic voltammetry (CV) analysis}

Figure 1a shows the plot of capacitive performances for PACN composites at different scan rates $(5,10,20,30,50,60,80$ and $100 \mathrm{mV} / \mathrm{s})$ in a threeelectrode configuration with applied potential. The capacitive performance was characterized by cyclic voltammetry $(\mathrm{CV})$ in $1(\mathrm{M}) \mathrm{KCl}$ solution to obtain the value of specific capacitance $\left(C_{\mathrm{sp}}\right)$ of the composites. The cathodic reduction and the anodic oxidation process are the reason behind the development of negative and positive current in the cyclic voltamogram. In general, the ideal capacitor behavior of the composites was shown when ideal rectangular shape of the cyclic voltamogram was observed. However, PACN composite shows deviation of the ideality of the curve in Figure 1a which concludes its pseudo-capacitive nature. The reason behind the deviation from the ideality of the curve is that the interaction between electrode and electrolyte is reduced [29]. The specific capacitance of the PACN composites can be estimated using Equation (1) [27], the obtained specific capacitance value of PACN composite is $\approx 834 \mathrm{~F} / \mathrm{g}$ at a constant scan rate of $5 \mathrm{mV} / \mathrm{s}$. This high specific capacitance value of the PACN composites was obtained due to the contributions of both components such as PANI and $\mathrm{CNH}$ in the composites. In the PACN composites, PANI coated $\mathrm{CNH}$ increased the intimate interaction between them (PANI and $\mathrm{CNH}$ ) that facilitate to enhance the charge transfer among the components with high electrical conductivity. Thus, the effective charge transfers in the electrode takes part a crucial role to enhance the specific capacitance value as well as rate capability of the composites [30, 31]. The well-ordered nanostructure in the PACN composites can reduce the ionic diffusion path, facilitate ionic motion to the inner part, and improve utilization of electrode materials. Effective anchoring of the $\mathrm{CNH}$ on the PANI helps for easy and fast ion transport in the electrode material and increases the capacitance value of the composites. Moreover, the additional Faradaic charge transfer involved at the electrodeelectrolyte interface in the presence of $\mathrm{CNH}$ which also plays a key role to enhance the specific capacitance value in the PACN composites. Figure $1 \mathrm{~b}$ shows the capacitive performances plot for pure PANI. The specific capacitance value for pure PANI is $\approx 231 \mathrm{~F} / \mathrm{g}$ at $5 \mathrm{mV} / \mathrm{s}$ and Figure $1 \mathrm{c}$ shows specific 

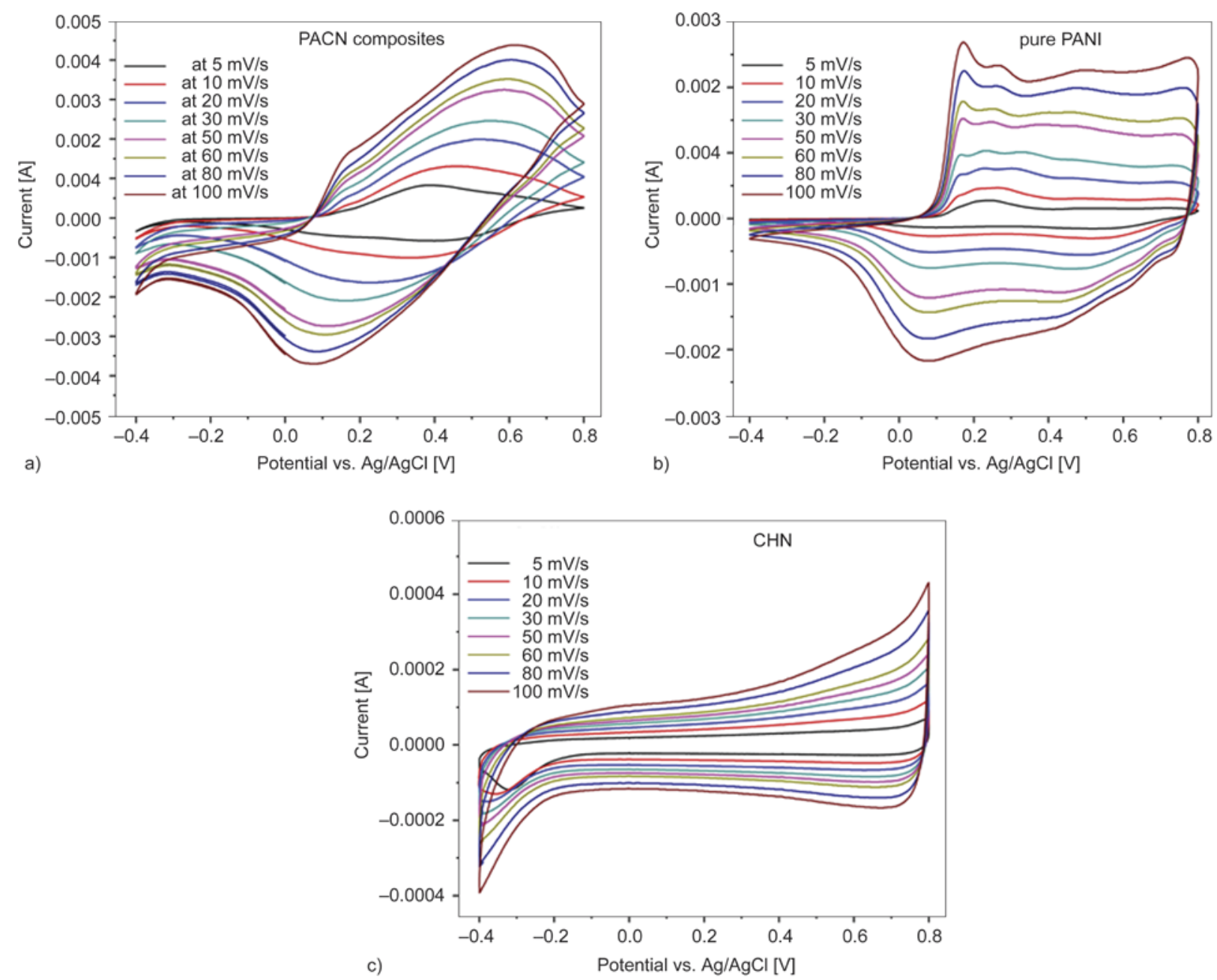

Figure 1. Cyclic voltammogram of (a) PACN composites, (b) pure PANI and (c) $\mathrm{CNH}$ at different scan rates

capacitance curve of $\mathrm{CNH}$ and capacitance value of $\approx 145 \mathrm{~F} / \mathrm{g}$ was obtained at same scan rate of $5 \mathrm{mV} / \mathrm{s}$ for $\mathrm{CNH}$. Many researchers $[32,33]$ have reported that the capacitance value of the composites can increase due to the deposition of metal oxides or metal salts $\left[\mathrm{RuO}_{2}\right.$ or $\left.\mathrm{Ni}(\mathrm{OH})_{2}\right]$ on reduced graphene oxide (RGO) sheets or by preparing thin films of PANI fibers and RGO sheets [34]. This high capacitance value of the composites is obtained due to contributions of the conducting RGO sheets and the pseudo-capacitance of the metal oxides or conducting polymer.

Figure 2 shows the variation of specific capacitance of the PACN composites and pure PANI with scan rate. Cyclic voltammetry of the PACN composites and pure PANI was done at various scan rates of 5 , $10,20,30,50,60,80$ and $100 \mathrm{mV} / \mathrm{s}$. From the figure, it is clearly seen that the specific capacitance value of the PACN composites and PANI strongly depends on the scan rate and it decreases with increasing the scan rate [2]. As can be seen, the spe-

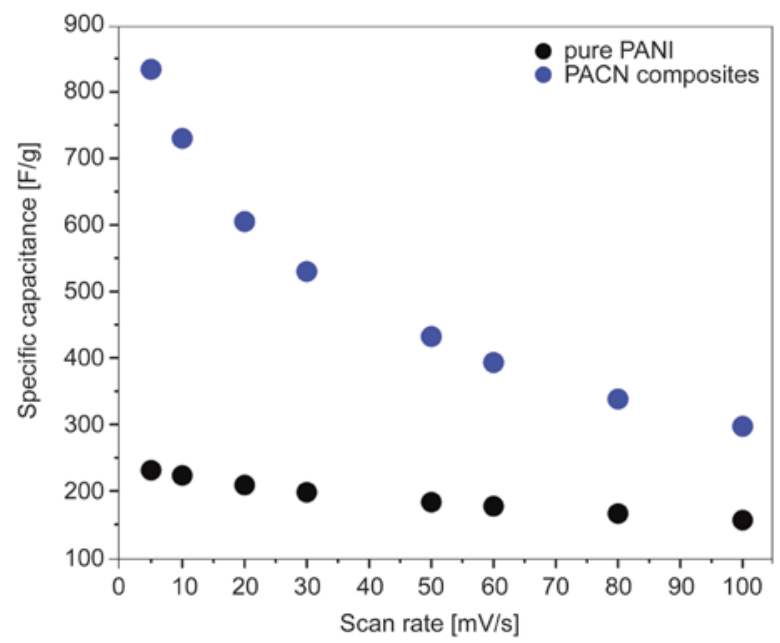

Figure 2. Plot of Specific capacitance vs. Scan rate for PACN composites and pure PANI

cific capacitance of the composites strongly depends on the scan rate and it gradually decreases for the PACN composites at a higher scan rate, as shown in Figure 2. At high scan rate, high diffusion resistance and large electrochemical polarization affect the 
electrode material which reduced the capacitive behavior of the composites [35]. This suggested the high degree of sustainability of capacitance even at a higher scan rate. The coating of PANI on $\mathrm{CNH}$ increases the $\pi-\pi$ stacking interaction between $\mathrm{CNH}$ and PANI chains, which may facilitate the electron transfer and plays a synergistic effect to the electrochemical properties of the hybrid composites [36]. This may be attributed to decreasing internal resistance as compared to the PANI electrode.

\subsubsection{Constant current charging/discharging (CCD) analysis}

The constant current charging/discharging (CCD) analysis of the PACN composites is shown in Figure 3 which is performed at a constant current density of $5 \mathrm{~A} / \mathrm{g}$. A triangular shaped symmetric charge/ discharge curve is obtained for PACN composites which unambiguously indicate the ideal capacitive behavior of the composites. The specific capacitance $\left(C_{\mathrm{sp}}\right)$ value of the PACN composites was calculated from the CCD curve using Equation (7) [37]:

$$
C_{\mathrm{sp}}=\frac{I \cdot \Delta t}{\Delta V \cdot m}
$$

where $I$ represents applied current, $\Delta V$ signifies the voltage and $\Delta t$ stands for average time in seconds and $m$ is the mass of the PACN composites, respectively. Thus, the calculated specific capacitance value of the PACN composites is $\approx 857 \mathrm{~F} / \mathrm{g}$.

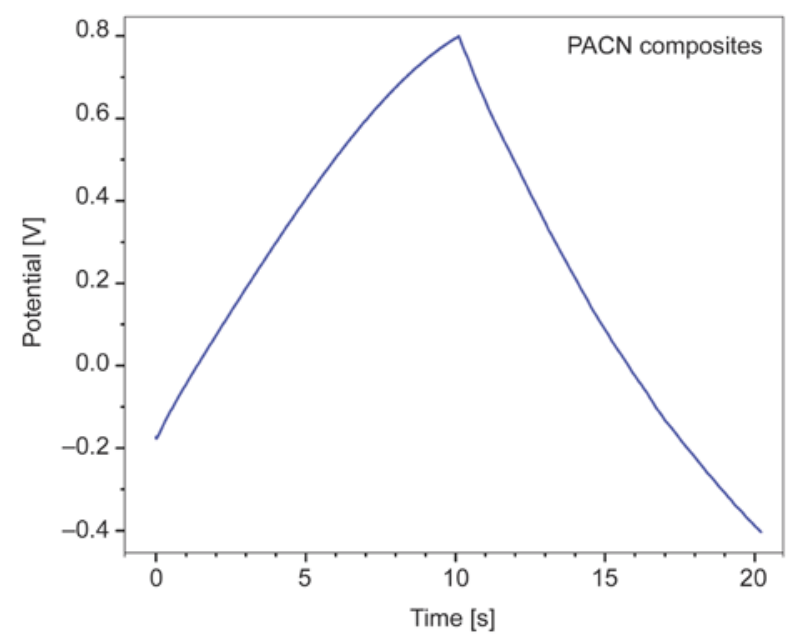

Figure 3. Cyclic charge/discharge curve of PACN composites

\subsubsection{Cycle-life stability test}

The variation of specific capacitance of PACN composites with cycle number is shown in Figure 4. The cycle stability of the composites has been estimated through repeating the cyclic voltammetry $(\mathrm{CV})$ in $1(\mathrm{M}) \mathrm{KCl}$ electrolyte and it is carried out at a scan rate of $10 \mathrm{mV} / \mathrm{s}$ for 500 cycles. As observed, the PACN composites remain $84 \%$ specific capacitance value after 500 cycles. In addition, the specific capacitance value of the PACN composites gradually decreases with increasing cycle number. Cho et al. also studied the electrochemical performance of PANI thin films. They have also measured the cyclic stability up to 500 cycles [38]. The $\pi$ - $\pi$ interaction and intermolecular interaction among the PANI and $\mathrm{CNH}$ resist the changing of regular network structure in the PACN composites and help to improve the cyclic stability of PACN composites. Thus, the regular network structure plays a key role in the PACN composites for enhancing the cyclic stability.

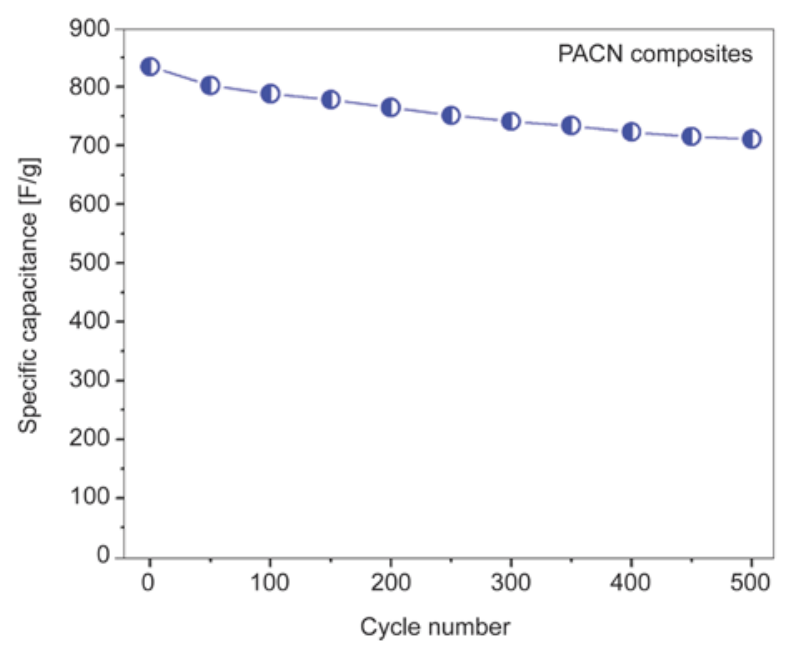

Figure 4. Specific capacitance vs. cycle number of PACN composites in $1(\mathrm{M}) \mathrm{KCl}$ solution

\subsubsection{Electrochemical impedance spectroscopic (EIS) analysis}

The Nyquist plot of impedance and their fitting circuit for the PACN composites are shown in Figure 5. The electrochemical impedance behavior of the composites is known from the Nyquist plot of impedance which is performed in the frequency region from $100 \mathrm{mHz}$ to $10 \mathrm{MHz}$ (Figure 5a). The Nyquist plot of impedance represents two components, one 
is the real component $\left(Z^{\prime}\right)$ and other is imaginary component $\left(Z^{\prime \prime}\right)$. The real component $\left(Z^{\prime}\right)$ of the composites gives ohmic properties and the imaginary component $\left(Z^{\prime \prime}\right)$ stands for the capacitive properties [39]. An ideal supercapacitor will show three frequency dependent regions in the Nyquist plot [40]. The supercapacitor behaves like a pure resistor at high frequency region. At medium frequency region, the electrode porosities can be observed. The electrolyte penetrates deeper and deeper into the porous structure of the electrode for decreasing the frequency from the very high frequency region and resulted a huge numbers of electrode surface for ion adsorption. The medium frequency region in the Nyquist plot of impedance is well known as Warburg curve. The low frequency region of the Nyquist plot shows the pure capacitive behavior. At low frequency region, the imaginary part sharply increases and a vertical line is obtained. However, ideal supercapacitor in real world is impossible. Solution resistance $\left(R_{\mathrm{S}}\right)$ of the composites can be obtained from the intercepts in highest frequency of the curves at real axis. The depressed semicircle at the higher frequency region in the Nyquist plots indicates the charge transfer resistance $\left(R_{\mathrm{ct}}\right)$. Warburg resistance $(W)$ of the composites is observed at intermediate frequency region. Generally, the constant phase element (CPE) is used to express the non-ideal behavior of the supercapacitor and it is denoted by ' $n$ '

a)
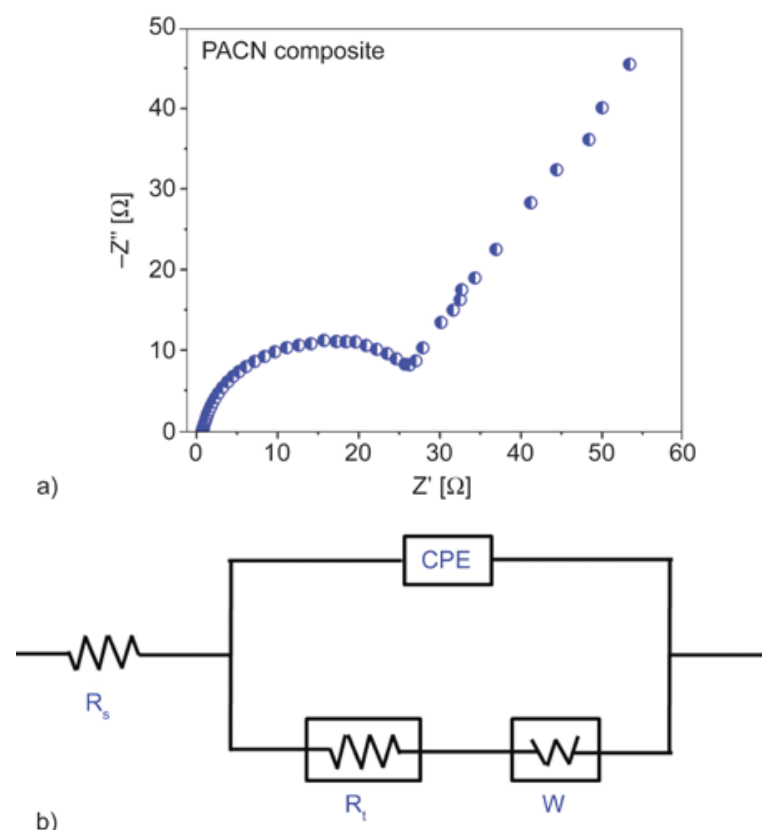

Figure 5. Nyquist plot (a) and equivalent electrical circuit (b) used in EIS fitting data of PACN composites value [41]. The Nyquist plots were analyzed by fitting the experimental impedance spectra to an equivalent electrical circuit. Thus, Figure $5 \mathrm{~b}$ shows a suitable fitting circuit for PACN composites which is schematically drawn. As observed, semicircle is seen at high frequency region and a straight line is shown at the low frequency range. The $\pi-\pi$ interaction among the PANI and CNH plays a crucial role to help for efficient electrolyte accessibility to the electrode surface through shortening the ion diffusion path. The observed vertical line at low frequency region indicates the good capacitive behavior and low diffusion resistance of the electrode material. The quality of the electrode materials strongly depends on the ' $n$ ' value. The supercapacitor will be called ideal when $n$ value will be equal to 1 . If ' $n$ ' value is zero, then it will be called insulators. In the case of PACN composites, obtained $\mathrm{n}$ value is $0.83(>0.5)$ which indicates the moderate capacitor behavior.

\subsection{Morphology study}

Figure 6 shows the morphology of the pure PANI, $\mathrm{CNH}$ and PACN composites. Figure 6a represents the FESEM image of PANI which indicates the fiber like structure of the PANI. The FESEM image of the $\mathrm{CNH}$ is shown in Figure 6b. From this image (Figure 6b), the flower like image of $\mathrm{CNH}$ is clearly seen. Figure $6 \mathrm{c}$ and $6 \mathrm{~d}$ indicate the high magnification FESEM images of PACN composites. From these images, fiber like structure of PACN composites is clearly observed where $\mathrm{CNH}$ is coated with the PANI. This provides more active sites for nucleation and electron transfer path and helps to increase the $\pi-\pi$ interaction with PANI in the composites. Thus, the specific capacitance of the PACN composites is enhanced due to the successful coating of $\mathrm{CNH}$ and formation of fiber like PACN composites. Figure $6 \mathrm{e}$ and $6 \mathrm{f}$ indicate the HRTEM images of PACN composites at different magnifications. Figure $6 \mathrm{e}$ indicates the formation of PANI-coated fiber like structure of $\mathrm{CNH}$ in PACN composites. The successful coating of $\mathrm{CNH}$ is clearly observed in Figure $6 \mathrm{f}$. Thus, the fiber like structure of the composites plays a crucial role to improve the specific capacitance value of the PACN composites. From the both FESEM and HRTEM images, it is clearly seen that $\mathrm{CNH}$ are homogeneously dispersed and formed a conducting network path in the composites which is also responsible for the higher specific capacitance 

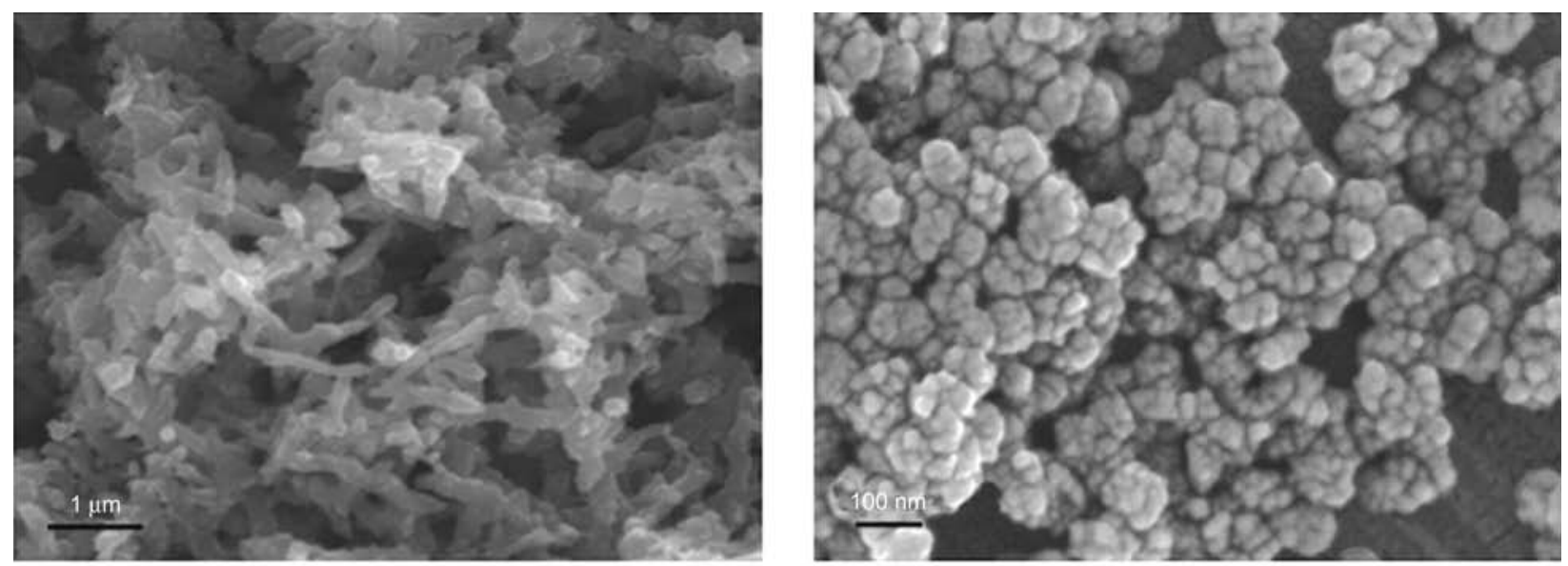

a)

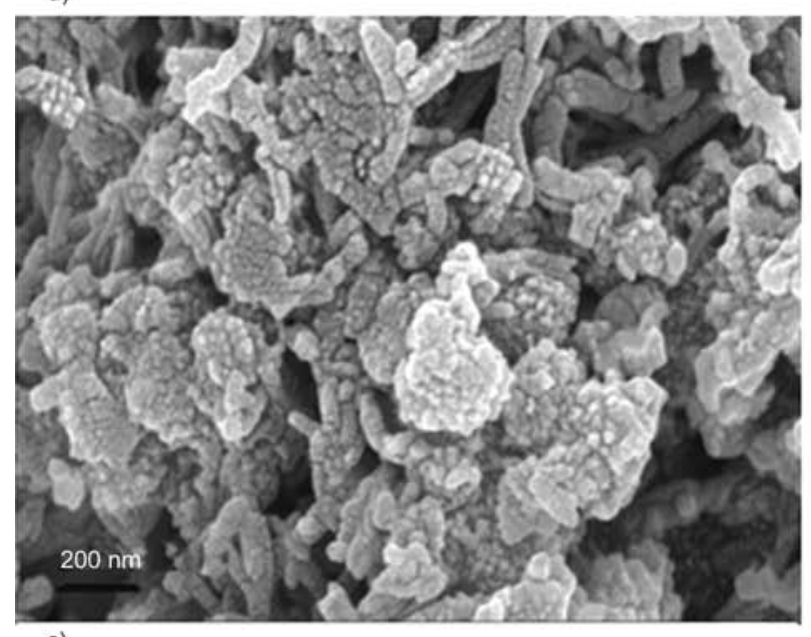

c)

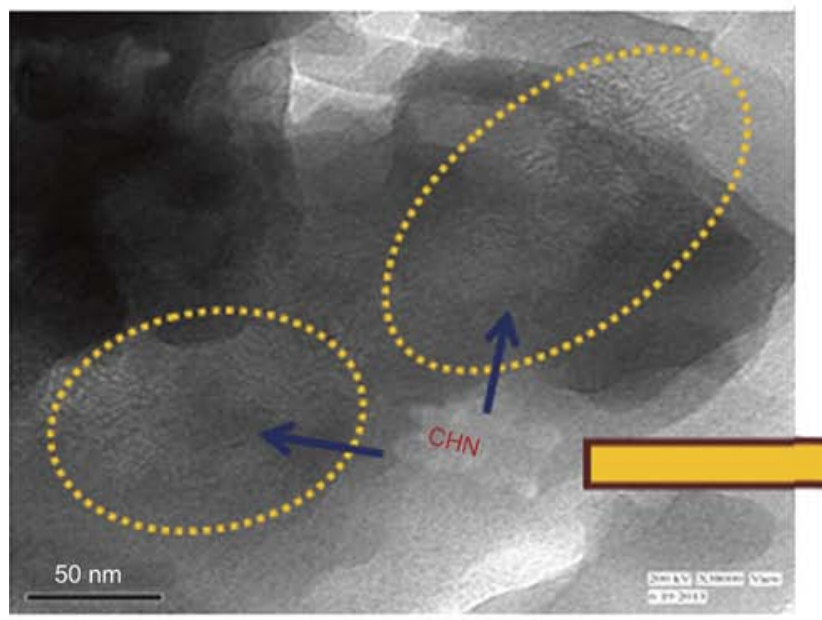

e)

b)

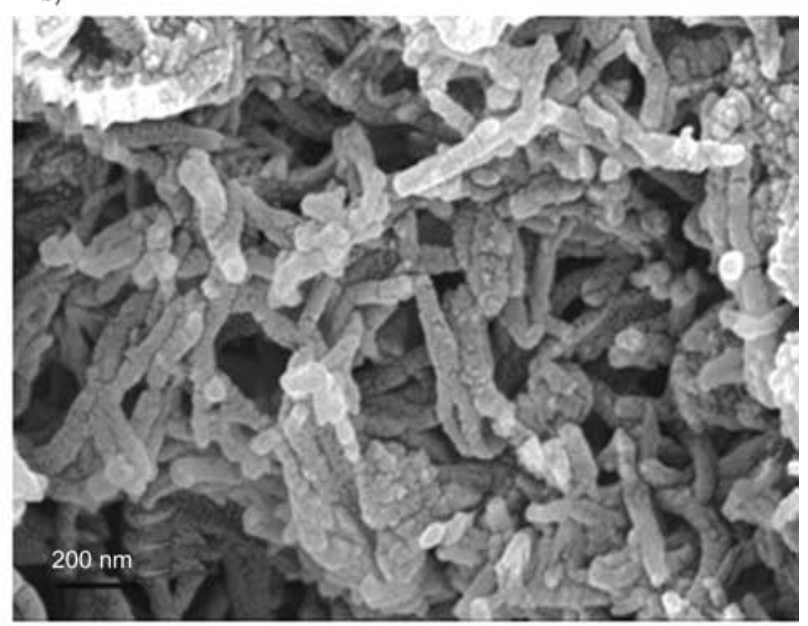

d)

Figure 6. FESEM micrographs of (a) pure PANI, (b) pure CNH and (c, d) PACN composites at two different magnifications. HRTEM micrographs of $(e, f)$ of PACN composites at two different magnifications

value as well as conducting behavior of the PACN composites.

\subsection{Comparative study}

Several researchers have already reported the specific capacitance values for different composites based on PANI or other composites. Feng et al. [42] have obtained specific capacitance value of $\approx 640 \mathrm{~F} / \mathrm{g}$ for PANI/graphene composites films which were synthesized through in-situ polymerization of aniline in the presence of graphite oxide (GO). The composites show $90 \%$ retention life after 1000 charge/ discharge cycles. Wang et al. [43] have synthesized PANI/flexible graphene hybrid materials via in-situ 
polymerization-reduction/dedoping-redoping process. The reported specific capacitance value of the composites was $\approx 1126 \mathrm{~F} / \mathrm{g}$. Li et al. [44] have prepared PANI nanofiber/graphene nanosheets composites through in-situ polymerization in $1(\mathrm{M}) \mathrm{H}_{2} \mathrm{SO}_{4}$ solution. The composites show specific capacitance value of $\approx 1130 \mathrm{~F} / \mathrm{g}$ at $5 \mathrm{mV} / \mathrm{s}$ scan rate with retention of $87 \%$ specific capacitance after 1000 harge/discharge processes. Liu et al. [45] have achieved the specific capacitance value of $\approx 301 \mathrm{~F} / \mathrm{g}$ for PANI nanofibers/ flexible graphene sheet composites. Zhang et al. [46] has synthesized PANI doped graphene composites and reported value of specific capacitance was $\approx 480 \mathrm{~F} / \mathrm{g}$ at current density of $0.1 \mathrm{~A} / \mathrm{g}$. Wang et al. [47] have reported gravimetric capacitance of $\approx 233 \mathrm{~F} / \mathrm{g}$ and volumetric capacitance of $\approx 135 \mathrm{~F} / \mathrm{cm}^{3}$ for PANI/flexible graphene composites. The composites have been synthesized by in-situ electropolymerization method. Sahoo et al. [48] have synthesized polypyrrole (PPy) nanofiber/graphene composites through in-situ polymerization and obtained specific capacitance value of the composites was $\approx 466 \mathrm{~F} / \mathrm{g}$ at $10 \mathrm{mV} / \mathrm{s}$ scan rate. Hu et al. [49] have synthesized highly porous nanorod-PANI/graphene composites films through in-situ electrochemical polymerization method. They have obtained specific capacitance value of $\approx 878.57 \mathrm{~F} / \mathrm{g}$ at a current density of $1 \mathrm{~A} / \mathrm{g}$. Yan et al. [50] have achieved specific capacitance of $\approx 637 \mathrm{~F} / \mathrm{g}$ at $2 \mathrm{mV} / \mathrm{s}$ scan rate for $\mathrm{MnO}_{2}$ based composites which was prepared through coating of amorphous $\mathrm{MnO}_{2}$ onto crystalline $\mathrm{SnO}_{2}$ nanowires, grown on stainless steel substrate. Cong et al. [51] have synthesized PANI nanorods/flexible graphene composites papers through in-situ electropolymerization technique. Thus, prepared PANI nanorods/flexible graphene composites show specific capacitance value of $\approx 763 \mathrm{~F} / \mathrm{g}$ with good cycling stability. Feng et al. [52] prepared $\mathrm{MnO}_{2} /$ graphene composites by hydrothermal method and reported specific capacitance of $\approx 516.8 \mathrm{~F} / \mathrm{g}$ at a scan rate of $1 \mathrm{mV} / \mathrm{s}$. Fan et al. [53] synthesized polyaniline hollow spheres (PANI-HS) electrochemically reduced graphene oxide (ERGO) hybrids with core-shell structures through solution-based co-assembly process. The specific capacitance of hybrid composites was $\approx 614 \mathrm{~F} / \mathrm{g}$ at a current density of $1 \mathrm{~A} / \mathrm{g}$. Qian et al. [54] prepared CNT/PPy core/shell composites via $\pi-\pi$ interaction. The reported specific capacitance of the composites was $\approx 276.3 \mathrm{~F} / \mathrm{g}$ at current density of $1 \mathrm{~A} / \mathrm{g}$. Chang et al. [55] have syn- thesized PANI/MWCNT nanocomposites films using fresh plant leaves as a template through the nanocasting technique. The obtained specific capacitance of the nanocomposites was $\approx 535 \mathrm{~F} / \mathrm{g}$ at a current density of $1 \mathrm{~A} / \mathrm{g}$ for $5 \mathrm{wt} \%$ MWCNT loading. In our study, we have achieved high specific capacitance value of $\approx 834 \mathrm{~F} / \mathrm{g}$ for PACN composites at $5 \mathrm{mV} / \mathrm{s}$ scan rate compared to most of the above reported methods.

\subsection{Electrical properties \\ 4.4.1. DC electrical conductivity}

The PACN composites show high DC electrical conductivity $\left(\sigma_{\mathrm{DC}}\right)$ in the order of $\approx 6.7 \cdot 10^{-2} \mathrm{~S} / \mathrm{cm}$. This very high electrical conductivity of the composites can be explained considering the formation of continuous conductive interconnected network structure of $\mathrm{CNH}-\mathrm{CNH}$ and $\mathrm{CNH}-\mathrm{PANI}$ in the PACN composites even at exceptionally low $\mathrm{CNH}$ content. This high electrical conductivity of the PACN composites is well supported by high capacitance value. Generally, the electrical conduction is generated in polymer composites through tunneling of charge carriers between the conducting nanofillers. The changing of the conducting value in the conducting polymer composites occurred due to tunneling conduction [56]. The energy barrier change in the composites strongly depends on the nature of the matrix polymer and the fabrication method. The current in a tunnel junction varied with the barrier width and exponentially decreases with it. If the nanofillers are randomly distributed in the polymer composites, then the barrier width is considered the mean average distance $(d)$ between the conducting nanofillers and in the first approximation, it is directly proportional to the nanofillers concentration in weight, $p^{-1 / 3}[57]$. So, the tunneling conductivity $\left(\log \sigma_{\mathrm{DC}}\right)$ can be expressed by Equation (8):

$\log \left(\sigma_{\mathrm{DC}}\right) \propto p^{-1 / 3}$

In general, the tunneling occurs among the adjacent nanofillers in the composites. Generally, the electrons in the polymer composites cannot shift from one electrode to another electrode due to existence of high energy barrier insulating or less conductive matrix polymer. However, the energy barrier gap is reduced by applying of voltage from the source between the two electrodes which act as a driving force for the movement of electrons by tunneling con- 
duction. Thus, the electrons in the composites are moved and enhanced the electrical conductivity of the composites. In addition, $\pi$ - $\pi$ interaction between $\mathrm{CNH}-\mathrm{CNH}$ and CNH-PANI help to reduce the contact resistance and enhanced the electrical conductivity of the PACN composites.

\subsubsection{AC electrical conductivity}

Figure 7 shows the variation of AC electrical conductivity $\left(\sigma_{\mathrm{AC}}\right)$ with frequency $(f)$ of the PACN composites which is measured in the frequency range of $\sim 10^{1}$ to $\sim 10^{6} \mathrm{~Hz}$ at room temperature. As observed, the value of $\sigma_{\mathrm{AC}}$ depends on the frequency and it increases with the increase in frequency. The value of $\sigma_{\mathrm{AC}}$ remains almost same up to certain frequency, known as critical frequency $\left(f_{\mathrm{c}}\right)$ and after that (above $f_{\mathrm{c}}$ ), electrical conductivity suddenly increased with frequency $[58,59]$. The value of $\sigma_{\mathrm{AC}}$ of any dielectric material below fc (at low frequency range) is the summation of two components and can be written by Equation (9):

$\sigma_{\mathrm{AC}}=\sigma_{\mathrm{DC}}+\omega \varepsilon^{\prime \prime}$

where $\sigma_{\mathrm{DC}}$ is the DC electrical conductivity, $\omega$ represents angular frequency which is equal to $2 \pi f$ and $\varepsilon^{\prime \prime}$ stands for dielectric loss factor.

The $\sigma_{\mathrm{DC}}$ is the first component of the above equation which develops due to the ionic or electronic conductivity. The second component $\left(\omega \varepsilon^{\prime \prime}\right)$ of the above equation varies with the extent of polarization of dipoles (induced and permanent) and accumulated interfacial charges which are well known as Maxwell-Wagner-Sillars (MWS) effect. At low

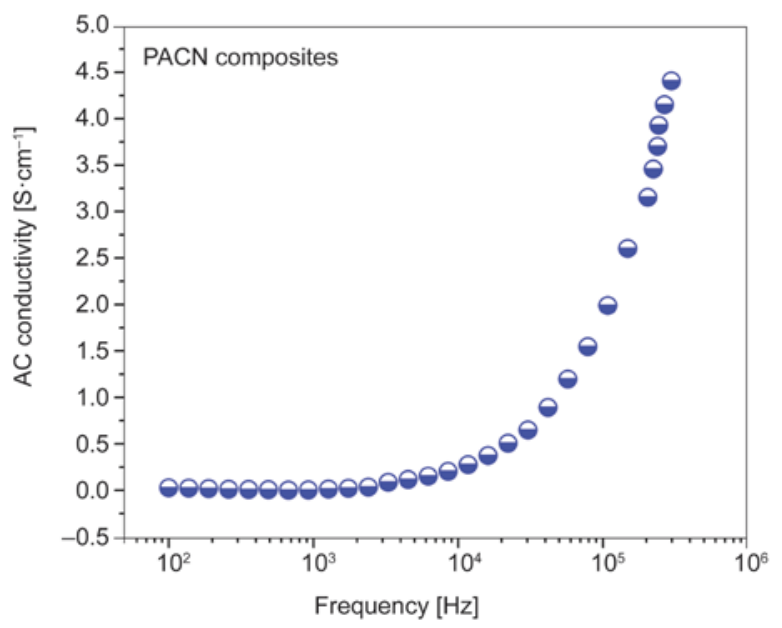

Figure 7. AC conductivity vs. frequency of the PACN composites frequency region (below $f_{\mathrm{c}}$ ), the effect of interfacial polarization becomes more significant and the dipoles/induced dipoles get enough time to orient themselves with the direction of applied electric field. So, the value of $\sigma_{\mathrm{AC}}$ actually indicates the $\sigma_{\mathrm{DC}}$ for a conductive system at low frequency region.

The polarization effect becomes insignificant at high frequency region (above $f_{\mathrm{c}}$ ) and the dipoles do not get enough relaxation time to orient them in the direction of applied electric field. The applied AC electric field (periodic alternation) above $f_{\mathrm{c}}$ results in the radical reduction of space charge accumulation and dispersion of dipoles in the applied field direction that reduces the extent of polarization. Thus, the value of $\sigma_{\mathrm{AC}}$ varies strongly with the excitation of the charge particles and electrons move through the continuous interconnected conducting network path in the composites. Additionally, it can be assumed that the hopping of excited electrons through the inter particle gap (thin polymer layer) becomes easier above $f_{\mathrm{c}}$, adding to the conductivity that already exists at low frequency in the composites.

\section{Conclusions}

In this work, PANI coated $\mathrm{CNH}$ based composites (PACN) has been successfully synthesized as a supercapacitor materials by in-situ polymerization method. The composites show high specific capacitance value $(\approx 834 \mathrm{~F} / \mathrm{g}$ at $5 \mathrm{mV} / \mathrm{s})$ compared to the pure PANI $(\approx 231 \mathrm{~F} / \mathrm{g}$ at $5 \mathrm{mV} / \mathrm{s})$ and $\mathrm{CNH}$ $(\approx 145 \mathrm{~F} / \mathrm{g}$ at $5 \mathrm{mV} / \mathrm{s})$. Thus, cost-effective and simple method for the preparation of PACN composite gives high capacitance value which is the major success of this study. The very high aspect ratio of $\mathrm{CNH}$ plays a crucial role to achieve high capacitance value of the composites. The $\pi$ - $\pi$ interaction among the electron rich phenyl rings of PANI and conducting $\mathrm{CNH}$ nanofiller in the PACN composites also take part to improve the specific capacitance value and the electrical conductivity of the composites than that of pure PANI. Thus, the obtained high specific capacitance value of the PACN composites indicated that this composite can be used as promising materials for supercapacitor applications. In addition, the PACN composite is electrically conductive in nature. So, it can also be used in the various conducting field of applications. The capacitive behavior, morphological study and electrical properties of the PACN composites were thoroughly characterized. 


\section{Acknowledgements}

The author acknowledges CSIR, New Delhi, India for their financial support.

\section{References}

[1] Cuentas-Gallegos A. K., Lira-Cantú M., Casañ-Pastor N., Gómez-Romero P.: Nanocomposite hybrid molecular materials for application in solid-state electrochemical supercapacitors. Advanced Functional Materials, 15, 1125-1133 (2005).

DOI: $10.1002 / \mathrm{adfm} .200400326$

[2] Miller J. R., Simon P.: Electrochemical capacitors for energy management. Science, 321, 651-652 (2008).

DOI: $10.1126 /$ science. 1158736

[3] Jang B. Z., Liu C., Neff D., Yu Z., Wang M. C., Xiong W., Zhamu A.: Graphene surface-enabled lithium ionexchanging cells: Next-generation high-power energy storage devices. Nano Letters, 11, 3785-3791 (2011). DOI: $10.1021 / \mathrm{nl} 2018492$

[4] Kim T. Y., Lee H. W., Stoller M., Dreyer D. R., Bielawski C. W., Ruoff R. S., Suh K. S.: High-performance supercapacitors based on poly(ionic liquid)modified graphene electrodes. ACS Nano, 5, 436-442 (2010). DOI: $10.1021 / \mathrm{nn} 101968 \mathrm{p}$

[5] Khilari S., Pandit S., Ghangrekar M. M., Pradhan D., Das D.: Graphene oxide-impregnated PVA-STA composite polymer electrolyte membrane separator for power generation in a single-chambered microbial fuel cell. Industrial and Engineering Chemistry Research, 52, 11597-11606 (2013).

DOI: $10.1021 / \mathrm{ie} 4016045$

[6] Long C., Wei T., Yan J., Jiang L., Fan Z.: Supercapacitors based on graphene-supported iron nanosheets as negative electrode materials. ACS Nano, 7, 1132511332 (2013). DOI: $10.1021 / \mathrm{nn} 405192 \mathrm{~s}$

[7] Li X-G., Huang M-R., Zeng J-F., Zhu M-F.: The preparation of polyaniline waterborne latex nanoparticles and their films with anti-corrosivity and semi-conductivity. Colloids and Surfaces A: Physicochemical and Engineering Aspects, 248, 111-120 (2004). DOI: $10.1016 /$ j.colsurfa.2004.08.077

[8] Huang M-R., Ding Y-B., Li X-G.: Combinatorial screening of potentiometric $\mathrm{Pb}$ (II) Sensors from polysulfoaminoanthraquinone solid ionophore. ACS Combinatorial Science, 16, 128-138 (2014).

DOI: $10.1021 / \operatorname{co} 400140 \mathrm{~g}$

[9] Huang M-R., Rao X-W., Li X-G., Ding Y-B.: Lead ion-selective electrodes based on polyphenylenediamine as unique solid ionophores. Talanta, 85, 15751584 (2011).

DOI: $10.1016 /$ j.talanta.2011.06.049

[10] Simon P., Gogotsi Y.: Materials for electrochemical capacitors. Nature Materials, 7, 845-854 (2008).

DOI: $10.1038 /$ nmat2297
[11] Zhang L. L., Zhao X. S.: Carbon-based materials as supercapacitor electrodes. Chemical Society Reviews, 38, 2520-2531 (2009). DOI: $10.1039 / \mathrm{B} 813846 \mathrm{~J}$

[12] Chen P-C., Shen G., Shi Y., Chen H., Zhou C.: Preparation and characterization of flexible asymmetric supercapacitors based on transition-metal-oxide nanowire/single-walled carbon nanotube hybrid thin-film electrodes. ACS Nano, 4, 4403-4411 (2010). DOI: $10.1021 / \mathrm{nn} 100856 \mathrm{y}$

[13] Khilari S., Pandit S., Das D., Pradhan D.: Manganese cobaltite/polypyrrole nanocomposite-based air-cathode for sustainable power generation in the singlechambered microbial fuel cells. Biosensors and Bioelectronics, 54, 534-540 (2014). DOI: $10.1016 /$ j.bios.2013.11.044

[14] Huang M-R., Ding Y-B., Li X-G.: Lead-ion potentiometric sensor based on electrically conducting microparticles of sulfonic phenylenediamine copolymer. Analyst, 138, 3820-3829 (2013). DOI: $10.1039 / \mathrm{c} 3 \mathrm{an} 00346 \mathrm{a}$

[15] Li X-G., Feng H., Huang M-R., Gu G-L., Moloney M. G.: Ultrasensitive $\mathrm{Pb}(\mathrm{II})$ potentiometric sensor based on copolyaniline nanoparticles in a plasticizer-free membrane with a long lifetime. Analytical Chemistry, 84, 134-140 (2012). DOI: $10.1021 / \mathrm{ac} 2028886$

[16] Li Y., Zhao X., Xu Q., Zhang Q., Chen D.: Facile preparation and enhanced capacitance of the polyaniline/ sodium alginate nanofiber network for supercapacitors. Langmuir, 27, 6458-6463 (2011). DOI: $10.1021 / 1 a 2003063$

[17] Chen D., Feng H., Li J.: Graphene oxide: Preparation, functionalization, and electrochemical applications. Chemical Reviews, 112, 6027-6053 (2012). DOI: $10.1021 / \mathrm{cr} 300115 \mathrm{~g}$

[18] Lee J. W., Hall A. S., Kim J-D., Mallouk T. E.: A facile and template-free hydrothermal synthesis of $\mathrm{Mn}_{3} \mathrm{O}_{4}$ nanorods on graphene sheets for supercapacitor electrodes with long cycle stability. Chemistry of Materials, 24, 1158-1164 (2012). DOI: $10.1021 / \mathrm{cm} 203697 \mathrm{w}$

[19] Zhu S., Xu G.: Single-walled carbon nanohorns and their applications. Nanoscale, 2, 2538-2549 (2010). DOI: $10.1039 / \mathrm{CONR} 00387 \mathrm{E}$

[20] Dai H., Zhang S., Hong Z., Li X., Xu G., Lin Y., Chen G.: Enhanced photoelectrochemical activity of a hierarchical-ordered $\mathrm{TiO}_{2}$ mesocrystal and its sensing application on a carbon nanohorn support scaffold. Analytical Chemistry, 86, 6418-6424 (2014).

DOI: $10.1021 / \mathrm{ac} 500813 \mathrm{u}$

[21] Izadi-Najafabadi A., Yamada T., Futaba D. N., Yudasaka M., Takagi H., Hatori H., Iijima S., Hata K.: Highpower supercapacitor electrodes from single-walled carbon nanohorn/nanotube composite. ACS Nano, 5, 811-819 (2011). DOI: $10.1021 / \mathrm{nn} 1017457$ 
[22] Wu Q., Xu Y., Yao Z., Liu A., Shi G.: Supercapacitors based on flexible graphene/polyaniline nanofiber composite films. ACS Nano, 4, 1963-1970 (2010).

DOI: $10.1021 / \mathrm{nn} 1000035$

[23] Deshmukh A. B., Shelke M. V.: Synthesis and electrochemical performance of a single walled carbon nanohorn- $\mathrm{Fe}_{3} \mathrm{O}_{4}$ nanocomposite supercapacitor electrode. RSC Advances, 3, 21390-21393 (2013).

DOI: 10.1039/C3RA43079K

[24] Wang D., Wang Q., Wang T.: Morphology-controllable synthesis of cobalt oxalates and their conversion to mesoporous $\mathrm{Co}_{3} \mathrm{O}_{4}$ nanostructures for application in supercapacitors. Inorganic Chemistry, 50, 6482-6492 (2011).

DOI: 10.1021/ic200309t

[25] Kumar N. A., Choi H-J., Shin Y. R., Chang D. W., Dai L., Baek J-B.: Polyaniline-grafted reduced graphene oxide for efficient electrochemical supercapacitors. ACS Nano, 6, 1715-1723 (2012).

DOI: $10.1021 / \mathrm{nn} 204688 \mathrm{c}$

[26] Xu J., Wang K., Zu S-Z., Han B-H., Wei Z.: Hierarchical nanocomposites of polyaniline nanowire arrays on graphene oxide sheets with synergistic effect for energy storage. ACS Nano, 4, 5019-5026 (2010).

DOI: $10.1021 / \mathrm{nn} 1006539$

[27] Yan J., Wei T., Shao B., Fan Z., Qian W., Zhang M., Wei F.: Preparation of a graphene nanosheet/polyaniline composite with high specific capacitance. Carbon, 48, 487-493 (2010).

DOI: 10.1016/j.carbon.2009.09.066

[28] Bose S., Kuila T., Uddin M. E., Kim N. H., Lau A. K. T., Lee J. H.: In-situ synthesis and characterization of electrically conductive polypyrrole/graphene nanocomposites. Polymer, 51, 5921-5928 (2010).

DOI: 10.1016/j.polymer.2010.10.014

[29] Meher S. K., Justin P., Rao G. R.: Microwave-mediated synthesis for improved morphology and pseudocapacitance performance of nickel oxide. ACS Applied Materials and Interfaces, 3, 2063-2073 (2011). DOI: $10.1021 / \mathrm{am} 200294 \mathrm{k}$

[30] Jung H. J., Kim Y-J., Han J. H., Yudasaka M., Iijima S., Kanoh H., Kim Y. A., Kaneko K., Yang C-M.: Thermal-treatment-induced enhancement in effective surface area of single-walled carbon nanohorns for supercapacitor application. Journal of Physical Chemistry C, 117, 25877-25883 (2013).

DOI: $10.1021 / \mathrm{jp} 405839 \mathrm{z}$

[31] Dhibar S., Das C. K.: Silver nanoparticles decorated polyaniline/multiwalled carbon nanotubes nanocomposite for high-performance supercapacitor electrode. Industrial and Engineering Chemistry Research, 53, 3495-3508 (2014).

DOI: $\underline{10.1021 / \text { ie } 402161 \mathrm{e}}$
[32] Zhao D., Guo X., Gao Y., Gao F.: An electrochemical capacitor electrode based on porous carbon spheres hybrided with polyaniline and nanoscale ruthenium oxide. ACS Applied Materials and Interfaces, 4, 55835589 (2012).

DOI: $10.1021 / \mathrm{am} 301484 \mathrm{~s}$

[33] Sun Z., Lu X.: A solid-state reaction route to anchoring $\mathrm{Ni}(\mathrm{OH})_{2}$ nanoparticles on reduced graphene oxide sheets for supercapacitors. Industrial and Engineering Chemistry Research, 51, 9973-9979 (2012).

DOI: $10.1021 / \mathrm{ie} 202706 \mathrm{~h}$

[34] Gao Z., Yang W., Wang J., Wang B., Li Z., Liu Q., Zhang M., Liu L.: A new partially reduced graphene oxide nanosheet/polyaniline nanowafer hybrid as supercapacitor electrode material. Energy Fuels, 27, 568575 (2013). DOI: $10.1021 / \mathrm{ef301795g}$

[35] Zhang Y., Li G-Y., Lv Y., Wang L-Z., Zhang A-Q., Song Y-H., Huang B-L.: Electrochemical investigation of $\mathrm{MnO}_{2}$ electrode material for supercapacitors. International Journal of Hydrogen Energy, 36, 1176011766 (2011). DOI: 10.1016/j.ijhydene.2011.06.020

[36] Biswas S., Drzal L. T.: Multilayered nanoarchitecture of graphene nanosheets and polypyrrole nanowires for high performance supercapacitor electrodes. Chemistry of Materials, 22, 5667-5671 (2010). DOI: $10.1021 / \mathrm{cm} 101132 \mathrm{~g}$

[37] Wang Y., Shi Z., Huang Y., Ma Y., Wang C., Chen M., Chen Y.: Supercapacitor devices based on graphene materials. Journal of Physical Chemistry C, 113, 13103-13107 (2009). DOI: $10.1021 / j p 902214 \mathrm{f}$

[38] Cho S., Shin K-H., Jang J.: Enhanced electrochemical performance of highly porous supercapacitor electrodes based on solution processed polyaniline thin films. ACS Applied Materials and Interfaces, 5, 91869193 (2013). DOI: $10.1021 / \mathrm{am} 402702 \mathrm{y}$

[39] Chen W., Rakhi R. B., Hu L., Xie X., Cui Y., Alshareef H. N.: High-performance nanostructured supercapacitors on a sponge. Nano Letters, 11, 5165-5172 (2011). DOI: $10.1021 / \mathrm{nl} 2023433$

[40] Cheng Q., Tang J., Ma J., Zhang H., Shinya N., Qin L-C.: Polyaniline-coated electro-etched carbon fiber cloth electrodes for supercapacitors. Journal of Physical Chemistry C, 115, 23584-23590 (2011). DOI: $10.1021 / j p 203852$ p

[41] Liu J., An J., Ma Y., Li M., Ma R.: Synthesis of a graphene-polypyrrole nanotube composite and its application in supercapacitor electrode. Journal of Electrochemical Society, 159, A828-A833 (2012). DOI: $\underline{10.1149 / 2.093206 j e s}$ 
[42] Feng X-M., Li R-M., Ma Y-W., Chen R-F., Shi N-E., Fan Q-L., Huang W.: One-step electrochemical synthesis of graphene/polyaniline composite film and its applications. Advanced Functional Materials, 21, 29892996 (2011).

DOI: $10.1002 / \mathrm{adfm} .201100038$

[43] Wang H., Hao Q., Yang X., Lu L., Wang X.: A nanostructured graphene/polyaniline hybrid material for supercapacitors. Nanoscale, 2, 2164-2170 (2010). DOI: $10.1039 / \mathrm{c} 0 \mathrm{nr} 00224 \mathrm{k}$

[44] Li J., Xie H., Li Y., Liu J., Li Z.: Electrochemical properties of graphene nanosheets/polyaniline nanofibers composites as electrode for supercapacitors. Journal of Power Sources, 196, 10775-10781 (2011).

DOI: 10.1016/j.jpowsour.2011.08.105

[45] Liu S., Liu X., Li Z., Yang S., Wang J.: Fabrication of free-standing graphene/polyaniline nanofibers composite paper via electrostatic adsorption for electrochemical supercapacitors. New Journal of Chemistry, 35, 369-374 (2011). DOI: $10.1039 / \mathrm{C} 0 \mathrm{NJ} 00718 \mathrm{H}$

[46] Zhang D., Zhang X., Chen Y., Yu P., Wang C., Ma Y.: Enhanced capacitance and rate capability of graphene/ polypyrrole composite as electrode material for supercapacitors. Journal of Power Sources, 196, 5990-5996 (2011). DOI: 10.1016/j.jpowsour.2011.02.090

[47] Wang D-W., Li F., Zhao J., Ren W., Chen Z-G., Tan J., Wu Z-S., Gentle I., Lu G. Q., Cheng H-M.: Fabrication of graphene/polyaniline composite paper via in situ anodic electropolymerization for high-performance flexible electrode. ACS Nano, 3, 1745-1752 (2009). DOI: $10.1021 / \mathrm{nn} 900297 \mathrm{~m}$

[48] Sahoo S., Dhibar S., Hatui G., Bhattacharya P., Das C. K.: Graphene/polypyrrole nanofiber nanocomposite as electrode material for electrochemical supercapacitor. Polymer, 54, 1033-1042 (2013). DOI: 10.1016/j.polymer.2012.12.042

[49] Hu L., Tu J., Jiao S., Hou J., Zhu H., Fray D. J.: In situ electrochemical polymerization of a nanorod-PANIgraphene composite in a reverse micelle electrolyte and its application in a supercapacitor. Physical Chemistry Chemical Physics, 14, 15652-15656 (2012). DOI: $10.1039 / \mathrm{c} 2 \mathrm{cp} 42192 \mathrm{e}$

[50] Yan J., Khoo E., Sumboja A., Lee P. S.: Facile coating of manganese oxide on tin oxide nanowires with highperformance capacitive behavior. ACS Nano, 4, 42474255 (2010).

DOI: $10.1021 / \mathrm{nn} 100592 \mathrm{~d}$
[51] Cong H-P., Ren X-C., Wang P., Yu S-H.: Flexible graphene-polyaniline composite paper for high-performance supercapacitor. Energy and Environmental Science, 6, 1185-1191 (2013). DOI: $10.1039 / C 2 E E 24203 \mathrm{~F}$

[52] Feng X., Yan Z., Chen N., Zhang Y., Ma Y., Liu X., Fan Q., Wang L., Huang W.: The synthesis of shape-controlled $\mathrm{MnO}_{2}$ /graphene composites via a facile onestep hydrothermal method and their application in supercapacitors. Journal of Materials Chemistry A, 1, 12818-12825 (2013).

DOI: $10.1039 / \mathrm{C} 3 \mathrm{TA} 12780 \mathrm{~J}$

[53] Fan W., Zhang C., Tjiu W. W., Pramoda K. P., He C., Liu T.: Graphene-wrapped polyaniline hollow spheres as novel hybrid electrode materials for supercapacitor applications. ACS Applied Materials and Interfaces, 5, 3382-3391 (2013).

DOI: $10.1021 / \mathrm{am} 4003827$

[54] Qian T., Zhou X., Yu C., Wu S., Shen J.: Highly dispersed carbon nanotube/polypyrrole core/shell composites with improved electrochemical capacitive performance. Journal of Materials Chemistry A, 1, 15230-15234 (2013).

DOI: 10.1039/C3TA13624H

[55] Chang C-M., Weng C-J., Chien C-M., Chuang T-L., Lee T-Y., Yeh J-M., Wei Y.: Polyaniline/carbon nanotube nanocomposite electrodes with biomimetic hierarchical structure for supercapacitors. Journal of Materials Chemistry A, 1, 14719-14728 (2013).

DOI: 10.1039/C3TA13758A

[56] Gefen Y., Aharony A., Alexander S.: Anomalous diffusion on percolating clusters. Physical Review Letters, 50, 77-83 (1983).

DOI: 10.1103/PhysRevLett.50.77

[57] Bello A., Laredo E., Marval J. R., Grimau M., Arnal M. L., Müller A. J.: Universality and percolation in biodegradable poly( $\varepsilon$-caprolactone)/multiwalled carbon nanotube nanocomposites from broad band alternating and direct current conductivity at various temperatures. Macromolecules, 44, 2819-2828 (2011). DOI: $10.1021 / \mathrm{ma1} 02598 \mathrm{~h}$

[58] Dyre J. C.: The random free-energy barrier model for ac conduction in disordered solids. Journal of Applied Physics, 64, 2456-2468 (1988). DOI: $10.1063 / 1.341681$

[59] Dyre J. C., Schroder T. B.: Universality of ac conduction in disordered solids. Reviews of Modern Physics, 72, 873-892 (2000).

DOI: 10.1103/RevModPhys.72.873 